CASE REPORT

M. Adachi

\section{A Case of Varicella Zoster Virus Polyneuropathy: Involvement of the Glossopharyngeal and Vagus Nerves Mimicking a Tumor}

\begin{abstract}
SUMmARY: A 36-year-old woman presented with glossopharyngeal and vagus nerve palsy, which proved to be herpes zoster based on the high titers of Varicella zoster virus antibody in her serum. Thin-section T1-weighted images with contrast media demonstrated swelling and distinct contrast enhancement of the glossopharyngeal and vagus complex, mimicking a tumor. Following MR imaging, the size of the nerve complex returned to normal; however, the contrast enhancement remained longer than the symptoms.
\end{abstract}

V aricella zoster virus has a high affinity for the ganglion and causes various neuropathies including Ramsay Hunt syndrome. I was able to detect and follow abnormalities in the glossopharyngeal and vagus nerve (cranial nerves IX and X) complex in a patient with herpes zoster by using thin-section MR imaging with contrast media. Although many reports have documented the MR imaging findings of Ramsay Hunt syndrome, ${ }^{1-7}$ only a few have described abnormalities of cranial nerves IX and X. I present a series of MR images of abnormalities in the cranial nerve IX and X complex and discuss the association between these findings and the patient's symptoms.

\section{Case Report}

A 36-year-old woman had a sore throat on December 17, 2006. She consulted an otorhinologist and was prescribed drugs (600-mg ibuprofen and 1000-mg ampicillin daily). By December 19, her sore throat had worsened, and she noticed swelling of the neck lymph nodes. The following day she had trouble swallowing, and on the day after that, she had not only a slight fever and sore throat but also hoarseness. On December 25, a few blisters on the left aryepiglottic fold and paresis of the left soft palate were noticed. She was prescribed an antiherpetic drug (3000-mg valacyclovir hydrochloride daily) for 7 days (from December 25 to 31). On December 30, the fever, sore throat, and blisters on the aryepiglottic fold disappeared, and the paresis of the left soft palate and left vocal cord improved. By January 19, 2007, the swallowing disturbance and hoarseness had improved. By February 16, the paresis of the left vocal cord, hoarseness, and swallowing disturbance had disappeared completely.

Biochemical examination of blood obtained on December 19, 2006 , revealed the following values: white blood cell count, $5700 / \mu \mathrm{L}$; red blood cell count, 4,730,000/ $\mu \mathrm{L}$; lactate dehydrogenase, $208 \mathrm{IU} / \mathrm{L}$; and C-reactive protein, $<0.1 \mathrm{mg} / \mathrm{dL}$. In serum from blood obtained on December 25, 2006, and January 12, 2007, the titers of antibodies to Varicella zoster virus, immunoglobulin $\mathrm{G}$, and immunoglobulin $\mathrm{M}$ were significantly elevated.

To examine abnormalities of cranial nerves IX and X, I obtained MR images including T1-weighted images (TR, $520 \mathrm{~ms}$; TE, $15 \mathrm{~ms}$; FOV , $24 \times 24 \mathrm{~cm}$; section thickness, $7 \mathrm{~mm}$; section gap, $2 \mathrm{~mm}$; matrix,

Received February 15, 2008; accepted after revision March 3.

From the Department of Radiology, Ohshima Clinic, Yamagata, Japan.

Please address correspondence to Michito Adachi, MD, Department of Radiology, Ohshima Clinic, 4-1-14 Sakurada Nishi, Yamagata 990-2321, Japan; e-mail: miadchi@ beach.ocn.ne.jp

DOI 10.3174/ajnr.A1141
$512 \times 256$; NEX, 2); T2-weighted images (TR, $4000 \mathrm{~ms}$; TE, $102 \mathrm{~ms}$; FOV, $24 \times 24 \mathrm{~cm}$; section thickness, $7 \mathrm{~mm}$; section gap, $2 \mathrm{~mm}$; matrix, $512 \times 256$; NEX, 2; echo-train length, 12); routine T1-weighted images with contrast media (intravenous administration of 0.1 $\mathrm{mmol} / \mathrm{Kg}$ of gadopentetate dimeglumine, Magnevist; Bayer-Schering Pharma, Osaka, Japan); and thin-section T1-weighted images (1$\mathrm{mm}$-thick) with contrast media reformed by using 3D Fourier transformation spoiled gradient-recalled-echo (SPGR) images (TR, $30 \mathrm{~ms}$; TE, $4.3 \mathrm{~ms}$; flip angle, $35^{\circ}$; FOV, $18 \times 18 \mathrm{~cm}$; slab thickness, $64 \mathrm{~mm}$; matrix, $256 \times 256 \times 80$; NEX, 1) on December 26, 2006 and January 19, February 16, and March 13, 2007, respectively. The MR imaging study was performed by using a $1.5 \mathrm{~T}$ superconducting system (Signa Excite; GE Healthcare, Milwaukee, Wis) with an 8-channel neurovascular phased-array coil.

Although no abnormalities in the brain were detected on routine T1-weighted images, T2-weighted or T1-weighted images with contrast media, thin-section T1-weighted images with contrast media revealed abnormalities of cranial nerves IX and X. The nerve complex from the cisternal to the ganglionic region had thickened and had a distinct contrast enhancement resembling a tumor, such as a schwannoma (Fig 1). As time passed, the mass effect diminished and the contrast enhancement faded out; however, the enhancement remained longer than the symptoms (Figs 2-4). Abnormal contrast enhancement of the facial nerve or acoustic nerve was not seen in any MR imaging examination.

\section{Discussion}

Facial nerve palsy is well known as Ramsay Hunt syndrome and is caused by reactivation of the Varicella zoster virus. Patients with Ramsay Hunt syndrome typically present with a peripheral facial nerve palsy associated with sensorineural hearing loss, tinnitus, vertigo with nystagmus, and painful vesicular eruption. However, numerous articles have recently reported various cranial neuropathies, other than those of the facial and acoustic nerves. ${ }^{1,8-15}$ In particular, there have been many case reports of patients with glossopharyngeal and vagus nerve palsy caused by Varicella zoster virus infection, as in the present case. ${ }^{1,9-12,14,15}$ However, only a few have described the MR imaging findings of glossopharyngeal and vagus nerve palsy. ${ }^{1,15}$ Fortunately, I had the chance to depict and follow the abnormalities in the cranial nerve IX and X complex by using thin-section MR imaging with contrast media in a patient with Varicella zoster virus infection.

Reports of Ramsay Hunt syndrome have noted that the facial nerve under contrast enhancement might swell and resemble a small tumor. ${ }^{4-7}$ In the present case, when the symp- 


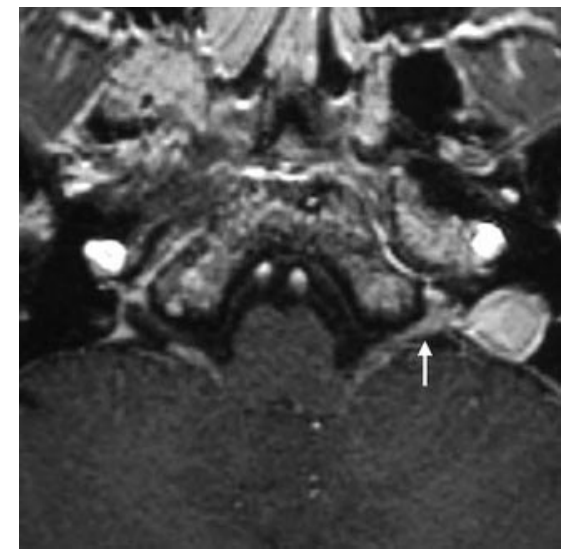

Fig 1. On a thin-section T1-weighted image (SPGR image) with contrast media obtained on December 26, 2006, the left glossopharyngeal and vagus nerve complex from the cisternal to ganglionic region is very thick and distinctly enhanced (arrow).

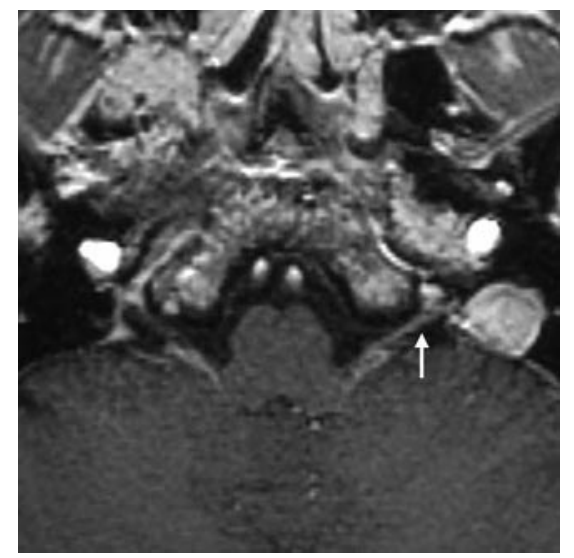

Fig 2. On a T1-weighted image obtained on January 19, 2007, the swelling of the nerve complex has diminished compared with the previous MR imaging study (arrow). The contrast enhancement continues.

toms were acute, the nerve complex also showed severe swelling. Previous histologic examination ${ }^{4}$ has revealed inflammatory infiltrates of lymphocytes and plasma cells in the geniculate ganglion of the facial nerve. In addition, intraneural edema in the neuronal nerve sheaths caused by breakdown of the blood-peripheral nerve barrier and by venous congestion in the epineural and perineural venous plexus was presumed. ${ }^{6,7}$ These inflammatory cells and edema most likely caused the swelling of the infected cranial nerves in the present patient.

In normal cranial nerves, contrast enhancement is not seen because of the blood-nerve barrier; however, in the first MR imaging study, cranial nerves IX and X had thickened, resembling a tumor (eg, a schwannoma) and had distinct contrast enhancement. As the mass effect of the nerve complex regressed, the symptoms improved; this change suggested some relationship between the swelling of the nerves and the symptoms. However, the contrast enhancement remained on the MR images obtained on February 16 and March 13, when the symptoms had already disappeared. A similar phenomenon of prolongation of contrast enhancement has previously been documented in cases of Ramsay Hunt syndrome and Bell palsy. ${ }^{7}$ The authors of that article suggested that no relationship could be established between the intensity of the enhancement

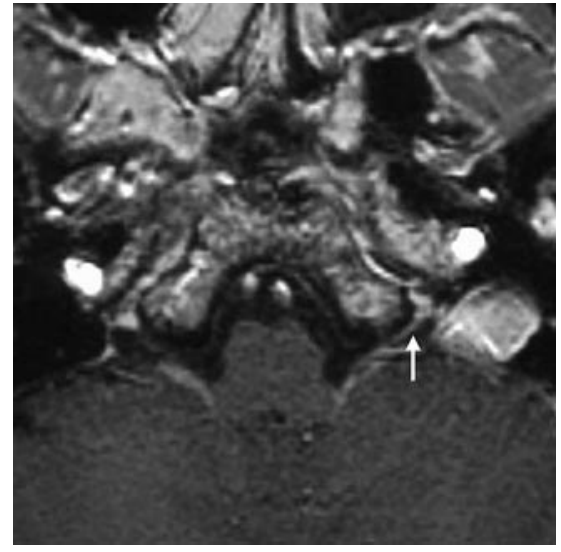

Fig 3. On a T1-weighted image obtained on February 16, 2007, the nerve complex has returned to normal size; however it is still distinctly enhanced (arrow).

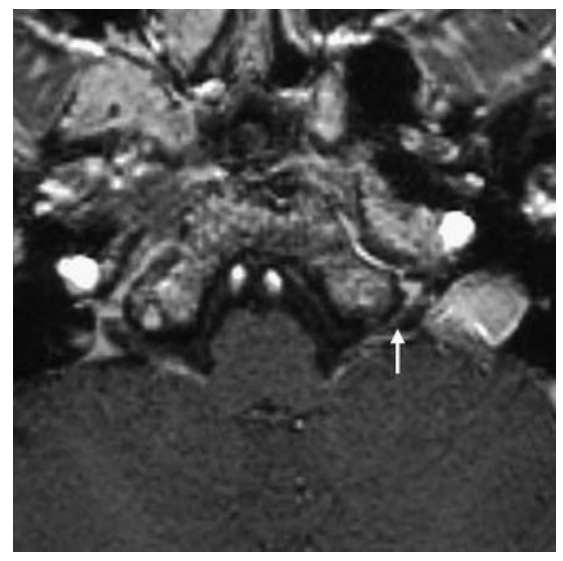

Fig 4. On a T1-weighted image obtained on March 13, 2007, the contrast enhancement of the nerve complex has almost disappeared but still remains (arrow).

and the prognosis for the facial palsy and that the long-lasting contrast enhancement was associated with a 2-phase breakdown in the blood-brain-nerve barrier consisting of breakdown of the endoneural barrier followed by breakdown of the perineurium. ${ }^{7}$ Therefore, swelling of the cranial nerves, not contrast enhancement, may be an indicator of the prognosis in neuropathies due to herpes zoster.

To the best of my knowledge, 2 case reports demonstrated the abnormalities of cranial nerves IX and $\mathrm{X}$ in patients with herpes zoster with MR imaging using contrast media. ${ }^{1,15}$ One depicted the liner and small nodular enhancement in cranial nerves IX and X and the ganglionic region, ${ }^{1}$ and the other report revealed a nodular lesion with contrast enhancement at the medial side of the left jugular foramen. ${ }^{15}$ These findings were somewhat similar to those of the present case when the symptoms were intense; however, unfortunately they did not offer a series of MR images following the abnormalities. The present case is very important for investigating the relationship between the MR imaging findings and symptoms.

\section{Conclusions}

I obtained a series of thin-section (1-mm-thick) T1-weighted images with contrast media following abnormalities in the glossopharyngeal and vagus nerve complex from infection by Varicella zoster virus. It may be that the natural course of 
reactivation of Varicella zoster virus is cranial nerve swelling when the symptoms are intense and prolonged contrast enhancement lasts longer than the symptoms.

\section{References}

1. Kikuchi H, Yoshimura T, Hara H, et al. A case of multiple cranial neuropathy due to varicella-zoster virus infection: detection on involvement of cranial ganglia with MRI [in Japanese]. Rinsho Shinkeigaku 1995;35:814-16

2. Naganawa S, Sugiura M, Kawamura M, et al. Prompt contrast enhancement of cerebrospinal fluid space in the fundus of the internal auditory canal: observations in patients with meningeal diseases on 3D-FLAIR images at 3 Tesla. Magn Reson Med Sci 2006;5:151-55

3. Suzuki F, Furuta Y, Ohtani F, et al. Herpes virus reactivation and gadoliniumenhanced magnetic resonance imaging in patients with facial palsy. Otol Neurotol 2001;22:549-53

4. Sartoretti-Schefer S, Kollias S, Valvanis A. Ramsay-Hunt syndrome associated with brain stem enhancement. AJNR Am J Neuroradiol 1999;20:278-80

5. Han MH, Jabour BA, Andrews JC, et al. Nonneoplastic enhancing lesions mimicking intracanalicular acoustic neuroma on gadolinium-enhanced MR images. Radiology 1991;179:795-96

6. Sartoretti-Schefer S, Wichmann W, Valavanis A. Gadolinium-enhanced MR in patients with idiopathic, herpetic, and HIV-associated facial nerve palsies: abnormal MR enhancement patterns. AJNR Am J Neuroradiol 1994; 15:479-85

7. Sartoretti-Schefer S, Brändle P, Wichmann W, et al. Intensity of MR contrast enhancement does not correspond to clinical and electroneurographic findings in acute inflammatory facial nerve palsy. AJNR Am J Neuroradiol 1996; 17:1229-36

8. Nagano K, Yoshimura K, Yamasaki M. A case of Ramsay Hunt syndrome associated with local meningitis, multiple cranial neuropathy, and the second cervical nerve involvement [in Japanese]. Rinsho Shinkeigaku 1999;39: 475-77

9. Miyazaki Y, Tajima Y, Sudo K, et al. A case of Ramsay Hunt syndrome initiated with hoarseness and dysphagia: consideration on spreading mechanisms of cranial neuropathy [in Japanese]. Rinsho Shinkeigaku 2002;42:855-58

10. Nishioka K, Fujishima K, Kobayashi H, et al. An extremely unusual presentation of varicella zoster viral infection of cranial nerves mimicking Garcin sydrome. Clin Neural Neuro Surg 2006;108:772-74. Epub 2005 Oct 13

11. Maeda A, Shoji T, Tsuchiya K, et al. A case of multiple cranial nerve palsy with severe dysphagia due to herpes zoster infection [in Japanese]. Rinsho Shinkeigaku 1992;32:524-26

12. Yoshioka A, Kitagawa Y, Kawada J, et al. A case of unilateral VIIth, IXth and Xth cranial nerve involvement with herpes zoster [in Japanese]. Rinsho Shinkeigaku 1990;30:413-15

13. Kobayashi Y, Riku S, Ieda T, et al. A case of herpes zoster associated with multiple cranial nerve involvement [in Japanese]. Rinsho Shinkeigaku 1992;32:314-16

14. Ohashi T, Fujimoto M, Shimizu H, et al. A case of isolated vagus nerve palsy with herpes zoster [in Japanese]. Rinsho Shinkeigaku 1994;34:928-29

15. Doi H, Segawa F, Koyano S, et al. A case of Vernet's syndrome due to varicellazoster virus infection [in Japanese]. Rinsho Shikeigaku 2001;41:695-97 